\title{
A UNITED-SET FORMULA
}

\author{
ZIV RAN ${ }^{1}$
}

Let a morphism $f: X \rightarrow Y$ of algebraic varieties be given. A united set or united $k$-tuple for $^{2}$ is a $k$-tuple $x_{1}, \ldots, x_{k}$ of distinct points on (or "infinitely near") $X$, such that $f\left(x_{1}\right)=\cdots=f\left(x_{k}\right)$. The purpose of this note is to announce an enumerative formula, valid under a restrictive hypothesis, for the united $k$-tuples of a map, i.e., a formula for the rational equivalence (or homology) class of a suitable cycle which parameterizes them. This yields as special cases formulas for the united $k$-tuples which contain a $k_{1}$-tuple, a $k_{2}$-tuple, etc. of mutually infinitely-near points. For our united- $k$-tuple cycle even to be defined, the morphism $f$ has to admit a certain kind of "resolution" (essentially it must factor through a "generic" map into a variety fibred by smooth curves over $Y$ ). Our result is sufficient, however, to yield formulas for the lines having prescribed contacts with a given projective variety having "generic" singularities and arbitrary dimension and codimension; these in turn yield formulas for the Thom-Boardman-Roberts singularity schemes [8] of a generic projection of such a variety. Classically such formulas were known for curves, for surfaces in $\mathbf{P}^{3}$, and in a few other cases, cf. [1]. Some recent results were obtained by Lascoux [6], Roberts [9] and LeBarz [7]. Our result yields new formulas even for surfaces in $\mathbf{P}^{4}$. For a modern account of these and related matters, see Kleiman's surveys [3, 5].

Admittedly, the hypothesis of existence of a "resolution" is a severe restriction on the morphism $f$. I am hopeful, however, that by pursuing further the same principles as in this paper, I will eventually obtain a united-set formula valid without such a restriction, and which would moreover be completely "intrinsic", in the sense of taking place on a suitable space associated solely to $X$ (which is not the case with the present formula).

We shall work in the category of complete (usually nonsingular) varieties over a field. Everything goes through with no change, however, in the category of compact complex manifolds.

1. Set-up. Fix a morphism $f: X \rightarrow Y$ of nonsingular varieties, and put $m=\operatorname{dim} X, n=\operatorname{dim} Y$. A resolution of $f$ is a diagram

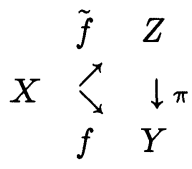

Received by the editors August 13, 1982.

1980 Mathematics Subject Classification. Primary 14E99; Secondary 14N10.

${ }^{1}$ Partially supported by NSF.

${ }^{2}$ This term is not consistent with the classical one of united point of a correspondence. 
where $\pi$ is a smooth morphism of relative dimension 1 , and $\tilde{f}$ is an embedding. More generally to define a $k$-quasi-resolution assume about $\tilde{f}$ only that its " $k$ 'fold locus" (cf. [4]), for all $k^{\prime}, 2 \leq k^{\prime} \leq k$, has codimension $>\left(k^{\prime}-1\right)(n-m)$ in $X$ (this is weaker than assuming that the latter locus has its expected codimension, which is $\left.\left(k^{\prime}-1\right)(n-m+1)\right)$. Now fix such a $k$-quasi-resolution. We will define some auxiliary objects. $Z^{k}$ is the $k$-fold fibre product of $Z$ over $Y ; Z_{X}^{k}$ is the fibre product $Z^{k-1} \times_{Y} X$, with coordinate projections $\pi^{k}: Z_{X}^{k} \rightarrow$ $Z^{k-1}, p^{k}: Z_{X}^{k} \rightarrow X ; j^{k}: Z_{X}^{k} \rightarrow Z^{k}$ is $\pi^{k} \times \tilde{f} ; \pi_{i}^{k}: Z_{X}^{k} \rightarrow Z$ is $\pi^{k}$ followed by the $i$ th coordinate projection; $q_{i}^{k}: Z_{X}^{k} \rightarrow Z_{X}^{k-1}$ is "delete the $i$ th coordinate". For $i<j \leq k$, the divisor $D_{i, j}^{k} \subset Z_{X}^{k}$ is defined by $\pi_{i}^{k}(\cdot)=\pi_{j}^{k}(\cdot)$; similarly, for $i<k, D_{i, k}^{k} \subset Z_{X}^{k}$ is defined by $\pi_{i}^{k}(\cdot)=p^{k}(\cdot)$; also put $D^{k}=\sum_{i=1}^{k-1} D_{i, k}^{k}$.

2. Formula. It is important to note that to each point $\mathrm{z}=\left(z_{1}, \ldots, z_{k-1}, x\right) \in$ $Z_{X}^{k}$ there corresponds a well-defined subscheme $\sigma(z)$ of $Z$ : namely this is the subscheme determined by the divisor $z_{1}+\cdots+z_{k-1}+\tilde{f}(x)$ on the smooth curve $\pi^{-1}\left(\pi\left(z_{1}\right)\right)$. Define the united $k$-tuple locus of $f$ as

$$
U_{k}(f)=\left\{\mathbf{z} \in Z_{X}^{k}: \sigma(\mathbf{z}) \subset \tilde{f}(X) \text { as schemes }\right\} .
$$

This definition is justified by the fact that the image of $U_{k}(f)$ in the Hilbert scheme of $X$ coincides, up to a lower-dimensional set, with the set of length- $k$ subschemes of $X$ which are mapped by $f$ to a single reduced point. The image of $U_{k}(f)$ in $X$ coincides with the " $k$-fold locus" of $f$ as defined by Kleiman [4].

Now the set $U_{k}=U_{k}(f)$ can (see $\left.\S 4\right)$ naturally be made into a cycle of "expected dimension" $k m-(k-1) n$ (i.e. expected codimension $(k-1)(n-m+1)$ in $Z_{X}^{k}$ ), and we seek a formula for the class $\left[U_{k}\right]$ of $U_{k}$ in the Chow group of $Z_{X}^{k}$ (though we could instead work in $Z^{k}$, working in $Z_{X}^{k}$ yields finer results). The result we get is an inductive one, and goes as follows.

THEOREM. Given a k-quasi-resolution as above, assume that $U_{k}$ and $U_{k-1}$ have their expected codimensions. Then if $k \geq 2$ we have

$$
\begin{aligned}
{\left[U_{k}\right]=} & \left(\pi^{k}\right)^{*}\left(j^{k-1}\right)_{*}\left(\left[U_{k-1}\right]\right) \\
& -\left(\sum_{i=1}^{k-1}\left(q_{i}^{k}\right)^{*}\left(\left[U_{k-1}\right]\right) \cdot\left[D_{i, k}^{k}\right]\right)\left\{\frac{\left(p^{k}\right)^{*}(c(\nu))}{1+\left[D^{k}\right]}\right\}_{n-m}
\end{aligned}
$$

in $C H^{(k-1)(n-m+1)}\left(Z_{X}^{k}\right)$ : where $\nu$ denotes the virtual normal bundle of $\tilde{f}$, i.e., $\tilde{f}^{*}(T Z)-T X, c(\nu)$ denotes its total Chern class, and \{\}$_{n-m}$ denotes the part in degree $n-m$. Also $U_{1}=1$.

3. Applications. By pushing the formula for $\left[U_{k}\right]$ down to $X$, we obtain a multiple-point formula à la Kleiman [4]. However, our formula contains more information than that. In particular, note that $U_{k} \cdot D_{i, j}^{k} \cdot D_{i^{\prime}, j^{\prime}}^{k} \cdots$ parametrizes those united $k$-tuples whose $i$ th and $j$ th, $i^{\prime}$ th and $j^{\prime}$ th, etc. points are infinitely near each other, so the theorem yields enumerative formulas for the united $k$ tuples which are the union of Thom-Boardman $S_{1}^{\left(q_{i}\right)}$-singularities, $i=1, \ldots, d$, $q_{1}+\cdots+q_{d}=k$, cf. Roberts [8]. 
I know two main types of maps which admit quasi-resolutions.

(a) Let $g: V \rightarrow \mathbf{P}^{N}$ be a map whose image has generic singularities (cf. [3]). Put $X=\left\{(v, L) \in V \times G\left(1, \mathbf{P}^{N}\right): g(v) \in L\right\}, Y=G\left(1, \mathbf{P}^{N}\right)$, and let $f: X \rightarrow Y$ be the projection, $Z \rightarrow Y$ the tautological $\mathbf{P}^{1}$ bundle, and $X \rightarrow Z$ the natural map. The united $k$-tuples of $f$ correspond to the $k$-secant lines of $g(V)$, and thus the theorem yields enumerative formulas for these. They include formulas for the $k$-secant lines having prescribed types of contact with $V$, as well as for the "varieties of contact" of such lines. For instance, if $V$ is a surface in $\mathbf{P}^{4}$, it will in general have a finite number of inflexional tangent lines meeting it elsewhere, say $L_{1}, \ldots, L_{r}$; a formula for $r$ was already given by LeBarz [7]. Put $L_{i} \cap V=3 p_{i}+q_{i}$. By pushing down to $V$ the formula for $\left.\left[U_{4}\right] \cdot\left[D_{1,2}^{4}\right] \cdot\left[D_{1,3}^{4}\right]\right)$, we get a formula for the rational equivalence class of $p_{1}+\cdots+p_{r}\left(\right.$ resp. $\left.q_{1}+\cdots+q_{r}\right)$ on $V$.

(b) Let $g: V \rightarrow \mathbf{P}^{N}$ be as above, and let $f: V \rightarrow Y=\mathbf{P}^{n}$ be $g$ followed by projection from a general center $M=\mathbf{P}^{N-n-1} \subset \mathbf{P}^{N}$, where $n \geq \operatorname{dim} V$. Then projection from a general codimension-1 subspace $M^{\prime} \subset M$ yields a quasiresolution $\tilde{f}: V \rightarrow \mathbf{P}^{n+1}$, so the theorem applies, yielding some formulas for the singularities of $f$, including those of Thom-Boardman-Roberts as above. Actually this case is a special case of case (a), because united points of $f$ correspond to $k$-secants of $\tilde{f}(V)$ passing through a fixed point.

4. Proof. As in other recent work on similar questions (see $[3,4,5]$ ), a key ingredient in the proof is an application of a "residual-intersection formula", of which we only require a relatively simple case, due to Fulton and MacPherson [2]. Consider the following cartesian diagram:

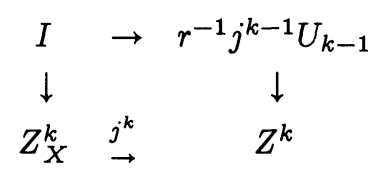

where $r: Z^{k} \rightarrow Z^{k-1}$ is projection onto the first $k-1$ coordinates. One can show that $I$ consists of $U_{k}$ plus a "residual" cycle, namely $\left(\pi^{k}\right)^{-1}\left(j^{k-1} U_{k-1}\right)$. $D^{k}$. Now [2] tells us how to compute the contribution of this residual cycle to the intersection-cycle $\left[r^{-1}\left(j^{k-1} U_{k-1}\right)\right] \cdot Z_{X}^{k}$, and this yields our formula.

ADDED IN PROOF. The hope expressed in the introduction is now a reality: a united-set formula taking place in a suitable "configuration space" $X^{[k]}$ and valid "modulo $\bar{S}_{2}(f)$ " has been obtained, as a consequence of a general formula for the rational-equivalence class of $V^{[k]}$ on $Z^{[k]}$, where $V \subset Z$ are arbitrary manifolds. As an application, among others, I obtain a formula for the class, in the moduli space of curves, of the locus of curves carrying a $g_{d}^{r}$, for given $r, d$.

ACKNOWLEDGEMENT. I would like to thank Professor Richard Fateman of the University of California, Berkeley, Computer Science Department who kindly gave me access to computer facilities and assisted me in their use, thus enabling me to obtain valuable experimental data. 


\section{REFERENCES}

1. H. F. Baker, Principles of geometry, vols. V, VI, Cambridge Univ. Press, New York, 1933.

2. W. Fulton and R. MacPherson, Intersecting cycles on an algebraic variety, Real and Complex Singularities (P. Holm (ed.)), Sijthoff \& Noordhoff, 1977, pp. 179-197.

3. S. L. Kleiman, The enumerative theory of singularities, ibid., pp. 297-396.

4. __, Multiple-point formulas. I: Iteration, Acta Math. 147 (1981), 13-49.

5. __ Multiple-point formulas for maps, Proc. Conf. Algebraic Geometry (Nice, 1981) (in press).

6. A. Lascoux, Calcul de certains polynômes de Thom, C. R. Acad. Sci. Paris Sér. A 278 (1974), 889-891.

7. P. LeBarz, Formules pour les multisécantes des surfaces, C. R. Acad. Sci. Paris Sér. A 292 (1981), 797-800.

8. J. Roberts, Singularity subschemes and generic projections, Trans. Amer. Math. Soc. 212 (1975), 229-268.

9. __ Some properties of double-point schemes, Compositio Math. 41 (1980), 61-94.

Department of Mathematics, Brandeis University, Waltham, MassACHUSETTS 02254 